\title{
FREE VIBRATION ANALYSIS OF FUNCTIONALLY GRADED BEAMS
}

\author{
Stanistaw Kukla, Jowita Rychlewska \\ Institute of Mathematics, Czestochowa University of Technology \\ Czestochowa, Poland \\ stanislaw.kukla@im.pcz.pl,jowita.rychlewska@im.pcz.pl
}

\begin{abstract}
In this paper free vibration of axially functionally graded (FG) beams consisting of two segments is studied. The FG beams under consideration are characterized by axially varying cross-section areas and/or functionally grading material properties. Numerical example for a beam that is clamped at both ends is presented.
\end{abstract}

Keywords: free vibration problem, functionally graded beams

\section{Introduction}

FG beams are characterized by uniaxially or spatially variable material properties. A list of papers on FG beams is very extensive. For example in [1] an analytical solution of a static cantilever functionally graded beam is formulated under the assumption that all the elastic moduli of the material have the same variations along the beam-thickness direction. Many papers deal with free and forced vibration analysis of FG beams, e.g. [2, 3]. For axially graded beams similar problems have become more complicated because of the governing equations with functional coefficients. We can mention here [4] where free vibration of exponentially graded beams is analyzed. Such problems are investigated also in [5] by using the Fredholm integral equations method. By expanding the mode shapes as power series, the resulting Fredholm equations were solved. In paper [6] free vibration and stability analysis of axially functionally graded tapered Timoshenko beams are carried out through a finite element approach.

In this contribution the formulation and solution of the vibration problem concerns the beams in the framework of Bernoulli-Euler theory. It is assumed that the changes of the cross-sectional area and material properties in the beam segments have an exponential form. The analytical solution of the problem is used for numerical analysis. The effect of selected parameters characterized the system on the free vibration frequencies of the clamped-clamped beam is investigated. 


\section{Formulation of the problem}

Consider a functionally graded beam of length $L$ (along the $x$ direction) consisting of two segments, having a non-uniform cross-section area $A(x)$, moment of inertia $I(x)$, modulus of elasticity $E(x)$ and material density $\rho(x)$. The equation of motion of the beam is given by [7]

$$
\frac{\partial^{2}}{\partial x^{2}}\left[E(x) I(x) \frac{\partial^{2} u}{\partial x^{2}}\right]+\rho(x) A(x) \frac{\partial^{2} u_{1}}{\partial t^{2}}=0
$$

where $u(x, t)$ is the transverse deflection of the beam at the position $x$ and in time $t$.

It is assumed that

$$
\begin{aligned}
& E(x) I(x)=\left\{\begin{array}{lll}
D_{1} e^{2 \beta_{1} \frac{x}{L}} & \text { for } & 0 \leq x \leq \eta L \\
D_{2} e^{2 \beta_{2} \frac{x}{L}} & \text { for } & \eta L x \leq L
\end{array}\right. \\
& \rho(x) A(x)=\left\{\begin{array}{lll}
m_{1} e^{2 \beta_{1} \frac{x}{L}} & \text { for } & 0 \leq x \leq \eta L \\
m_{2} e^{2 \beta_{2} \frac{x}{L}} & \text { for } & \eta L<x \leq L
\end{array}\right.
\end{aligned}
$$

where $0<\eta<1$ and $\beta_{1}, \beta_{2}$ are the dimensionless gradient parameters, $D_{1}, D_{2} . m_{1}, m_{2}$ are real constants. Substituting (2) into equation (1) and assuming that $u_{1}(x, t)=u(x, t)$ for $0 \leq x \leq \eta L$ and $u_{2}(x, t)=u(x, t)$ for $\eta L<x \leq L$, one obtains the governing equations of the beam vibrations in the form

$$
\begin{array}{ll}
\frac{\partial^{2}}{\partial x^{2}}\left[D_{1} e^{2 \beta_{1} \frac{x}{L}} \frac{\partial^{2} u_{1}}{\partial x^{2}}\right]+m_{1} e^{2 \beta_{1} \frac{x}{L}} \frac{\partial^{2} u_{1}}{\partial t^{2}}=0, & 0 \leq x \leq \eta L \\
\frac{\partial^{2}}{\partial x^{2}}\left[D_{2} e^{2 \beta_{2} \frac{x}{L}} \frac{\partial^{2} u_{2}}{\partial x^{2}}\right]+m_{2} e^{2 \beta_{2} \frac{x}{L}} \frac{\partial^{2} u_{2}}{\partial t^{2}}=0, & \eta L<x \leq L
\end{array}
$$

Using a separation of variables according to

$$
u_{1}(x, t)=U_{1}(x) \cos \omega t, \quad u_{2}(x, t)=U_{2}(x) \cos \omega t
$$

where $U_{i}(x), i=1,2$, are the corresponding amplitude functions and $\omega$ is the eigenfrequency of the beam. Substitution of equations (4) into equations (3) yields 


$$
\begin{array}{ll}
\frac{d^{2}}{d x^{2}}\left[D_{1} e^{2 \beta_{1} \frac{x}{L}} \frac{d^{2} U_{1}}{d x^{2}}\right]-m_{1} \omega^{2} L^{4} e^{2 \beta_{1} \frac{x}{L}} U_{1}=0, & 0 \leq x \leq \eta L \\
\frac{d^{2}}{d x^{2}}\left[D_{2} e^{2 \beta_{2} \frac{x}{L}} \frac{d^{2} U_{2}}{d x^{2}}\right]-m_{2} \omega^{2} L^{4} e^{2 \beta_{2} \frac{x}{L}} U_{2}=0, & \eta L<x \leq L
\end{array}
$$

Introducing the non-dimensional coordinate $\xi=\frac{x}{L}$ and dimensionless quantities

$$
\begin{aligned}
\Omega^{4}=\frac{m_{1}}{D_{1}} L^{4} \omega^{2}, & \mu=\sqrt{\frac{m_{2} D_{1}}{m_{1} D_{2}}}, \text { equations (5) can be written in the form } \\
& \frac{d^{2}}{d \xi^{2}}\left[e^{2 \beta_{1} \xi} \frac{d^{2} U_{1}}{d \xi^{2}}\right]-\Omega^{4} e^{2 \beta_{1} \xi} U_{1}=0, \quad 0 \leq \xi \leq \eta \\
& \frac{d^{2}}{d \xi^{2}}\left[e^{2 \beta_{2} \xi} \frac{d^{2} U_{2}}{d \xi^{2}}\right]-\mu^{2} \Omega^{4} e^{2 \beta_{2} \xi} U_{2}=0, \quad \eta<\xi \leq 1
\end{aligned}
$$

After some transformations we can rewrite equations (6) as follows

$$
\begin{aligned}
& \frac{d^{4} U_{1}}{d \xi^{4}}+4 \beta_{1} \frac{d^{3} U_{1}}{d \xi^{3}}+4 \beta_{1}^{2} \frac{d^{2} U_{1}}{d \xi^{2}}-\Omega^{4} U_{1}=0, \quad 0 \leq \xi \leq \eta \\
& \frac{d^{4} U_{2}}{d \xi^{4}}+4 \beta_{2} \frac{d^{3} U_{2}}{d \xi^{3}}+4 \beta_{2}^{2} \frac{d^{2} U_{2}}{d \xi^{2}}-\mu^{2} \Omega^{4} U_{2}=0, \quad \eta<\xi \leq 1
\end{aligned}
$$

Equations (7) are completed by boundary and continuity conditions. The continuity conditions are

$$
\begin{array}{ll}
U_{1}\left(\eta^{+}\right)=U_{2}\left(\eta^{-}\right) & \frac{d U_{1}}{d \xi}\left(\eta^{+}\right)=\frac{d U_{2}}{d \xi}\left(\eta^{-}\right) \\
\frac{d^{2} U_{1}}{d \xi^{2}}\left(\eta^{+}\right)=\frac{d^{2} U_{2}}{d \xi^{2}}\left(\eta^{-}\right) & \frac{d^{3} U_{1}}{d \xi^{3}}\left(\eta^{+}\right)=\frac{d^{3} U_{2}}{d \xi^{3}}\left(\eta^{-}\right)
\end{array}
$$

and the boundary conditions for clamped-clamped beam are

$$
\begin{array}{ll}
U_{1}(0)=0 & \frac{\partial U_{1}}{\partial \xi}(0)=0 \\
U_{2}(1)=0 & \frac{\partial U_{2}}{\partial \xi}(1)=0
\end{array}
$$




\section{Solution of the problem}

In this section a solution of the vibration problem (7)-(9) is presented. The general solution of equations (7) has the form

$$
\begin{array}{ll}
U_{1}(\xi)=e^{-\beta_{1} \xi}\left(C_{1} \cos \delta_{1} \xi+C_{2} \sin \delta_{1} \xi+C_{3} \cosh \delta_{2} \xi+C_{4} \sinh \delta_{2} \xi\right), & 0 \leq \xi \leq \eta \\
U_{2}(\xi)=e^{-\beta_{2} \xi}\left(C_{5} \cos \delta_{3} \xi+C_{6} \sin \delta_{3} \xi+C_{7} \cosh \delta_{4} \xi+C_{8} \sinh \delta_{4} \xi\right), & \eta<\xi \leq 1
\end{array}
$$

where

$$
\delta_{1}=\sqrt{\Omega^{2}-\beta_{1}^{2}}, \quad \delta_{2}=\sqrt{\Omega^{2}+\beta_{1}^{2}}, \quad \delta_{3}=\sqrt{\mu \Omega^{2}-\beta_{2}^{2}}, \quad \delta_{4}=\sqrt{\mu \Omega^{2}+\beta_{2}^{2}}
$$

Substituting functions (10) into (8) and (9) we obtain a set of equations with unknowns $C_{1}, \ldots, C_{8}$. Let us introduce the following denotations

$$
\begin{array}{ll}
m_{k i}=\left[\beta_{k}, \delta_{i}\right] \cdot \mathbf{v}_{i} & n_{k i}=\left[-\delta_{i}, \beta_{k}\right] \cdot \mathbf{v}_{i} \\
p_{k j}=\left[\beta_{k},-\delta_{j}\right] \cdot \mathbf{v}_{j} & r_{k j}=\left[-\delta_{j}, \beta_{k}\right] \cdot \mathbf{v}_{j} \\
\bar{m}_{k i}=\left[\left(\beta_{k}^{2}-\delta_{i}^{2}\right), 2 \beta_{k} \delta_{i}\right] \cdot \mathbf{v}_{i} & \bar{n}_{k i}=\left[-2 \beta_{k} \delta_{i},\left(\beta_{k}^{2}-\delta_{i}^{2}\right)\right] \cdot \mathbf{v}_{i} \\
\bar{p}_{k j}=\left[\left(\beta_{k}^{2}+\delta_{j}^{2}\right),-2 \beta_{k} \delta_{j}\right] \cdot \mathbf{v}_{j} & \bar{r}_{k j}=\left[-2 \beta_{k} \delta_{j},\left(\beta_{k}^{2}+\delta_{j}^{2}\right)\right] \cdot \mathbf{v}_{j} \\
\widetilde{m}_{k i}=\left[\left(3 \beta_{k} \delta_{i}^{2}-\beta_{k}^{3}\right),\left(-3 \beta_{k}^{2} \delta_{i}+\delta_{i}^{3}\right)\right] \cdot \mathbf{v}_{i} & \widetilde{n}_{k i}=\left[\left(3 \beta_{k}^{2} \delta_{i}-\delta_{i}^{3}\right),\left(3 \beta_{k} \delta_{i}^{2}-\beta_{k}^{3}\right)\right] \cdot \mathbf{v}_{i} \\
\widetilde{p}_{k j}=\left[\left(-3 \beta_{k} \delta_{j}^{2}-\beta_{k}^{3}\right),\left(3 \beta_{k}^{2} \delta_{j}+\delta_{j}^{3}\right)\right] \cdot \mathbf{v}_{j} & \widetilde{r}_{k j}=\left[\left(3 \beta_{k}^{2} \delta_{j}+\delta_{j}^{3}\right),\left(-3 \beta_{k} \delta_{j}^{2}-\beta_{k}^{3}\right)\right] \cdot \mathbf{v}_{j}
\end{array}
$$

where $\mathbf{v}_{i}=\left[\cos \delta_{i} \eta, \sin \delta_{i} \eta\right], \quad \mathbf{v}_{j}=\left[\cosh \delta_{j} \eta, \sinh \delta_{j} \eta\right], \quad k=1,2, \quad i=1,3, \quad j=2,4$. Under denotations (12) and $w=e^{\left(\beta_{1}-\beta_{2}\right) \eta}$ this set of equations can be written in the matrix form

$$
\mathbf{A}(\omega) \cdot \mathbf{C}=0
$$

where $\mathbf{C}=\left[C_{1} \ldots C_{8}\right]^{T}$ and

$$
\mathbf{A}=\left[\begin{array}{cccccccc}
1 & 0 & 1 & 0 & 0 & 0 & 0 & 0 \\
0 & \delta_{1} & 0 & \delta_{2} & 0 & 0 & 0 & 0 \\
0 & 0 & 0 & 0 & \cos \delta_{3} & \sin \delta_{3} & \cosh \delta_{4} & \sinh \delta_{4} \\
0 & 0 & 0 & 0 & -\delta_{3} \sin \delta_{3} & \delta_{3} \cos \delta_{3} & \delta_{4} \sinh \delta_{4} & \delta_{4} \cosh \delta_{4} \\
\cos \delta_{1} \eta & \sin \delta_{1} \eta & \cosh \delta_{2} \eta & \sinh \delta_{2} \eta & -w \cos \delta_{3} \eta & -w \sin \delta_{3} \eta & -w \cosh \delta_{4} \eta & -w \sinh \delta_{4} \eta \\
m_{11} & n_{11} & p_{12} & r_{12} & -w m_{23} & -w n_{23} & -w p_{24} & -w r_{24} \\
\bar{m}_{11} & \bar{n}_{11} & \bar{p}_{12} & \bar{r}_{12} & -w \bar{m}_{23} & -w \bar{n}_{23} & -w \bar{p}_{24} & -w \overline{2}_{24} \\
\widetilde{m}_{11} & \widetilde{n}_{11} & \widetilde{p}_{12} & \widetilde{r}_{12} & -w \widetilde{m}_{23} & -w \widetilde{n}_{23} & -w \widetilde{p}_{24} & -w \widetilde{r}_{24}
\end{array}\right]
$$


A non-trivial solution to equation (13) exists when the determinant of matrix $\mathbf{A}$ is equal to zero. Then the frequency equation of the beam vibration is

$$
\operatorname{det} \mathbf{A}(\omega)=0
$$

Equation (14) is then solved numerically.

\section{Numerical example}

In this section we present some numerical results. Table 1 shows the first four non-dimensional frequency parameters $\Omega_{i}(i=1,2,3,4)$ of the FG clamped-clamped beam for various values of parameters $\beta_{1}, \beta_{2}$ with fixed $\eta=0.5$ and $\mu=5$. It can be observed that an increase of the value of $\beta_{2}$ with fixed $\beta_{1}$ causes an increase of the frequency parameters $\Omega_{i}(i=1,2,3,4)$. Moreover, with an increase in $\beta_{1}$ ( $\beta_{2}$ is fixed) the frequency parameters $\Omega_{i}(i=1,2,3)$ are decreasing. The frequency parameter $\Omega_{4}$ changes non-monotonic. Diagrams of frequency parameter values $\Omega_{i}(i=1,2,3)$ as a functions of $\eta$ for $\beta_{1}=0.5, \beta_{2}=1$ and $\mu=1.75,2.75,4$, respectively, are presented in Figure 1 .

The first four non-dimensional free vibration frequencies for $\eta=0.5$ and $\mu=5$

\begin{tabular}{|c|c|c|c|c|}
\hline \multirow{4}{*}{-1} & -1 & 0 & 1 & 2 \\
\hline \multirow{4}{*}{$\beta_{2}$} & 2.47146 & 2.23620 & 1.90799 & 2.00000 \\
\cline { 2 - 5 } & 4.71453 & 4.54801 & 4.38694 & 4.23994 \\
\cline { 2 - 5 } & 7.04476 & 6.94461 & 6.86846 & 6.81638 \\
\cline { 2 - 5 } & 8.73202 & 8.67374 & 8.64946 & 8.66247 \\
\hline \multirow{4}{*}{0} & 2.62109 & 2.44218 & 2.22418 & 2.00000 \\
\cline { 2 - 5 } & 4.83775 & 4.68940 & 4.54715 & 4.41785 \\
\cline { 2 - 5 } & 7.11824 & 7.02212 & 6.95020 & 6.90233 \\
\cline { 2 - 5 } & 8.76384 & 8.70849 & 8.68687 & 8.70233 \\
\hline \multirow{4}{*}{1} & 2.77642 & 2.63385 & 2.47146 & 2.28476 \\
\cline { 2 - 5 } & 4.97371 & 4.84086 & 4.71453 & 4.60025 \\
\cline { 2 - 5 } & 7.20404 & 7.11210 & 7.04476 & 7.00159 \\
\cline { 2 - 5 } & 8.80375 & 8.75122 & 8.73202 & 8.74946 \\
\hline \multirow{3}{*}{2} & 2.94629 & 2.82991 & 2.70269 & 2.56454 \\
\cline { 2 - 5 } & 5.12346 & 5.00398 & 4.89131 & 4.79002 \\
\cline { 2 - 5 } & 7.30102 & 7.21334 & 7.15087 & 7.11293 \\
\cline { 2 - 5 } & 8.85185 & 8.80211 & 8.78498 & 8.80387 \\
\hline
\end{tabular}



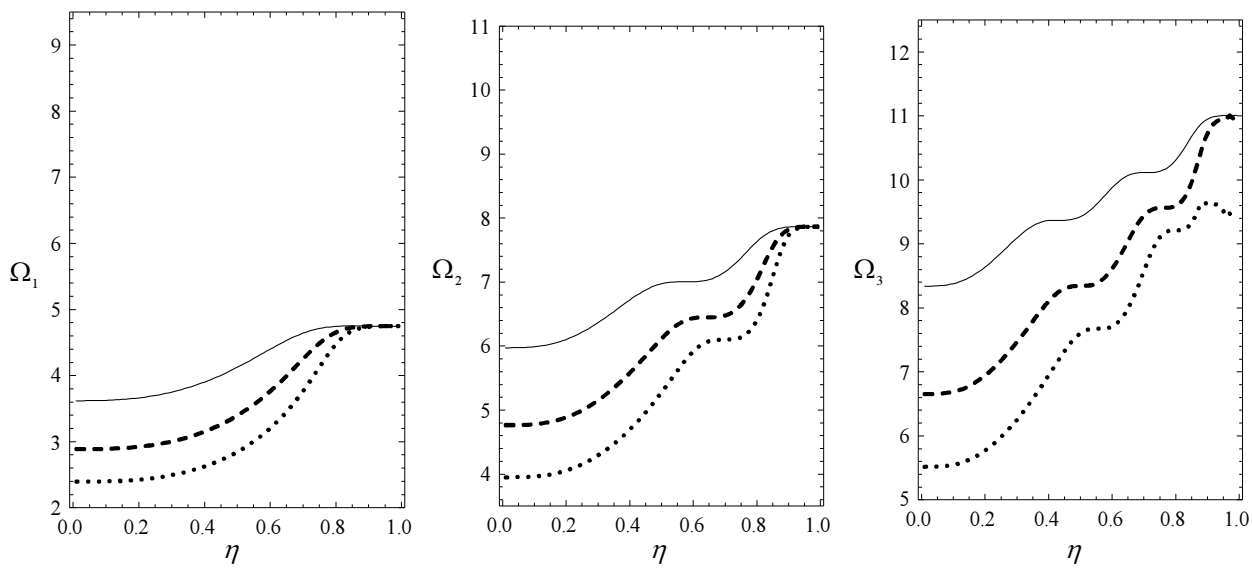

Fig 1. Frequency parameter values $\Omega_{i}$ for the first three modes of vibration as a function of $\eta$ for $\mu=1.75$ (solid line), $\mu=2.75$ (dashed line), $\mu=4$ (dotted line)

\section{Conclusions}

The free vibration problem of the FG beam consisting of two segments each of exponentially varying cross section areas and material properties is the subject of this paper. For a clamped-clamped beam the characteristic equation is derived. The numerical examples have shown the influence of gradient variation on the frequencies of the FG beam. The proposed approach can be applied to the vibration problem of beam consisting of an arbitrary number of segments.

\section{References}

[1] Zhong Z., Yu T., Analytical solution of a cantilever functionally graded beam, Compos. Sci. Technol. 2007, 67, 481-488.

[2] Trindade M.A., Benjeddou A., Refined sandwich model for vibration of beams with embedded shear piezoelectric actuators and sensors, Int. J. Comput. Struct. 2008, 86(9), 859-869.

[3] Aydogdu M., Taskin V., Free vibration analysis of functionally graded beams with simply supported edges, Mater. Design 2007, 28, 1651-1656.

[4] Li X.-F., Kang Y.-A., Wu J.-X., Exact frequency equations of free vibration of exponentially functionally graded beams, Appl. Acoustic 2013, 74, 413-420.

[5] Huang Y., Li X.-F., A new approach for free vibration of axially functionally graded beams with non-uniform cross-section, J. Sound. Vib. 2010, 329, 2291-2303.

[6] Shahba A., Attarnejad R., Marvi M.T., Hajilar S., Free vibration and stability analysis of axially functionally graded tapered Timoshenko beams with classical and non-classical boundary conditions, Compos. Part B Eng. 2011, 42, 801-808.

[7] Lebed O.I., Karnovsky I.A., Formulas for Structural Dynamics, Mc Graw-Hill, 2000. 